\title{
A Robust Algorithm for Estimation and Compensation of Doppler Shift in Underwater Acoustic OFDM Communication Systems
}

\author{
Thanika Reddy ${ }^{1}$, Vaishnavi Venkatesh ${ }^{2}$, Nupur Jain ${ }^{3}$ \\ Student, Dept of ECE, R.V. College of Engineering, Bangalore ${ }^{1,2,3}$
}

\begin{abstract}
Underwater acoustic communication has currently become a popular field of research. Underwater Acoustic Channels (UACs) are characterized by severe effects like multipath propagation, Doppler shift and scaling, intersymbol interference (ISI), frequency dependent attenuation and ocean noise. This paper addresses Doppler shift, in the presence of ocean noise and attenuation while using OFDM to transmit data. Frequency dependent attenuation, ocean noise and Doppler Effect have been simulated, on MATLAB (v2015a), to represent the UAC. At the receiver, a method to estimate and compensate this Doppler Effect has been developed. It uses two banks of parallel self-correlators for coarse followed by fine estimation and compensation. This method enables online receiver operation for multicarrier underwater acoustic communication. The same is verified on a Zynq board (ZC702).
\end{abstract}

Index Terms: Underwater acoustic channels, Orthogonal Frequency Division Multiplexing (OFDM), Doppler shift and scaling, Estimation and Compensation

\section{INTRODUCTION}

Underwater acoustic channels are characterized by various effects such as multipath, Doppler shift and scaling, attenuation, ocean noise, time variability and ISI. Doppler Effect is the shift in frequency and scaling of frequency due to the relative motion between the transmitter and receiver. The magnitude of Doppler scaling (a) is given as the ration between $v / c$, which is nothing but the relative speed of transmitter to the speed of sound (c). The speed of sound in water $(1500 \mathrm{~m} / \mathrm{s})$ which is very low when compared to the speed of electromagnetic waves in air. Therefore the effect of Doppler scaling is much lesser in air as compared to water and hence there is a greater need to estimate and compensate the Doppler Effect underwater. For example, let us take the case of a radio system at $160 \mathrm{~km} / \mathrm{h}(100 \mathrm{mph}), \mathrm{a}=1.5 \times 10^{-}$ 7. This value is low enough for us to ignore the effect of Doppler scaling. In other words, there is no need to estimate and compensate for it explicitly. The error is only $1 / 1000$ of a bit per 10,000 bits. In contrast to this situation, a stationary acoustic system may experience unintentional motion at $0.5 \mathrm{~m} / \mathrm{s}(1 \mathrm{knot})$, which would account for $\mathrm{a}=3 \times$ $10^{-4}$. For an AUV moving at several meters per second (submarines can move at much greater velocities), factor a will be of the order of $10^{-3}$, a value that cannot be ignored. The multicarrier modulation scheme OFDM is used in UWACs because of the advantages it offers. It can be easily implemented using DFT and IDFT operations [1], has very little overlap due to ISI [2] which can be eliminated using zero padding (ZP) or cyclic prefix (CP) as both offer similar BERs but each has an advantage of either lower power or scope for improvement of BER respectively [3], ICI is absent due to orthogonality of sub carriers [4], and it is robust to both time selective fading and frequency selective fading and also has high bandwidth efficiency and combats multipath interference [5]. Doppler effect shifts each subcarrier by a different amount [6]-[7], thus leading to a loss of orthogonality of subcarriers, crosstalk and reduced performance. Thus, it is vital to estimate and compensate Doppler effect. The M-ary digital modulation scheme used with OFDM affects the performance of the system and also determines the data rate. For UWACs, 4 QAM is shown to have lesser BER than 8 QAM and higher values of M [8]. A 64 point IDFT is used for with 16 carriers, to maintain Hermitian symmetry. The use of the Hermitian symmetry property of the DFT gives a real signal output, helps in reduction of peak-to-average power ratio (PAPR), and helps maintain same data rate with reduction in the modulation order compared to conventional OFDM systems that use $\mathrm{M}^{2}$ - ary QAM. This reduces PAPR further [9]. The nonlinear tolerance of OFDM systems is also increased using the Hermitian symmetry property of OFDM [10].

\section{LITERATURE SURVEY}

Underwater communication is one of the most difficult communication media as the relative motion between the transmitter and receiver is more significant underwater than terrestrial communication due to low speeds of sound in comparison to the speed of electromagnetic waves which leads to significant Doppler Effect [11]. As Doppler Effect causes both scaling and shift in frequency it is assumed, that path gains and Doppler rate are constant over the duration 
of the transmitter block. The receiver employs algorithms that rely on the preamble of a frame consisting of multiple OFDM symbols which estimate the Doppler scaling factor [12],[13].

Path loss and attenuation in a UWAC is caused due to two reasons. First is geometrical spreading, which models the increases in distance from the source and the expansion of the surface covered by the waves, with the travel of sound. Second is the conversion of acoustic energy to heat energy, which is dependent on frequency. These two reasons are considered in Thorp's model [14]. Also, the absorption coefficient has a complicated dependence on frequency. This dependence is simplified by the Thorp's attenuation model. Its validity is for frequencies below $50 \mathrm{kHz}$ [15], and the range of frequencies used in this project $(0.5 \mathrm{kHz}$ to $16.25 \mathrm{kHz})$ is well below $50 \mathrm{kHz}$.

Noise can either be site specific or ambient. Site specific noise is dependent on geography and location. For this reason, ambient noise from four sources is considered separately and summed up. The equation that approximates all four noises in is not considered because it is too optimistic (uses a no wave, no wind assumption) [14]. The model, along with a model of ambient ocean noise used in this project to characterize the channel, is discussed in [16].

The value of Doppler factor is given by the ratio of relative velocity between the transmitter and receiver and the speed of sound underwater [11]. AUVs with sensors travel with a speed of approximately $2 \mathrm{~m} / \mathrm{s}$ (6 knots) [17]. Assuming the receiver is stationary and the AUV is transmitting, this gives a value of 0.002 for Doppler scaling factor. Submarines travel at average speeds of $15 \mathrm{~m} / \mathrm{s}$ (30 knots). This gives a value of 0.01 for Doppler scaling factor. Using super cavitation, underwater vehicles have been developed which travel at speeds as high as $100 \mathrm{~m} / \mathrm{s}$ [18]. A value of 0.07 is obtained for this. Advances soon may lead to even higher speeds of underwater vehicles. Thus, to take all existing and upcoming speeds of underwater vehicles, the range of Doppler scaling factors used in this project is 0 to 0.099 .

The Doppler Effect manifests itself in two ways - it scales the duration of the symbol from T to T/ $(1+a)$, and it induces a frequency dependent shift $\mathrm{e}^{\mathrm{j} 22 \pi \mathrm{t}}$ in each subcarrier [19], where a is the Doppler scaling factor. The Doppler shift is thus modeled using this exponential term in the IDFT block of OFDM.

Various methods have been used in the past to estimate and compensate for the Doppler shift, the most obvious one being the adjustment of the carrier frequency at the receiver to compensate for the Doppler shift. Carrier synchronization can be done using the $\mathrm{M}^{\text {th }}$ law carrier recovery and/or a PLL [20]. Doppler causes each frequency component to shift by different amounts in wideband signals and this causes the Doppler Effect to be more accurately modeled using time scaling (expansion or compression) of the signal waveform $r(t)=s((1+\Delta) t)$, where $s(t)$ and $r(t)$ are the source signals and Doppler-shifted received signals respectively. Due to the high bandwidth of UAC (almost as high as an octave $(10-20 \mathrm{kHz}))$ the wideband model of the Doppler can be used. In this case, symbol synchronization and carrier synchronization are equally important. Conventional synchronization technique such as the coupling of PLLs with equalization is often unreliable in UAC, due to severe multipath distortion and fading [21].

Another method which is used for the estimation of the Doppler is done by using a LFM preamble and a LFM post amble around each data burst, this aids the receiver in the estimation of the change in the waveform duration. Resampling is performed after the Doppler scale estimate has been calculated. However, one of the major drawbacks associated with the method is that the entire data must be buffered before data demodulation, which prevents real time processing [21]. A few of the newer methods involve the transmission of two OFDM symbols that are identical along with a cyclic prefix, and the receiver uses a bank of parallel self-correlators [22]. The idea that the periodicity of the two symbols is maintained even after affected by Doppler, but periodicity changes, is used. Analytical results obtained provide with guidelines for the accurate selection of the Doppler scale resolution and detection threshold. Using just one preamble, similar performance of Doppler scale estimation and bit error rate is obtained in comparison to the method that uses two LFM waveforms. One of the major advantages of this method includes the ability to process in real time and the controllability of the accuracy of estimation.

Another method for estimation is a two-step method in which the search range of the ambiguity function used in correlators is first determined by analysis of the FFT spectrum of a continuous wave signal. This is a coarse estimation. Following this, the ambiguity function of an LFM signal is used in a bank of correlators for fine estimation of Doppler scaling factor [23]. This leads to a lesser number of correlators and greater accuracy of estimation. A similar method of estimation is based on the use of parallel self-correlators and cyclic prefix in the OFDM signal, where the Doppler shift estimation and compensation is done twice. This leads to a decrease in the number of self correlators because after the first estimation, it is comparatively simpler to implement the second bank of self correlators which helps in the reduction of complexity [24]. In this implementation, it is assumed that the Doppler scaling will remain constant for the entire duration of one frame.

\section{III.METHODOLOGY}

The order of design of the system is as follows. Simulation of OFDM Transmitter and Receiver, modelling Doppler Shift at Transmitter, incorporating estimation and two step compensation algorithm at receiver, and addition of Thorp's attenuation and ambient ocean noise is carrier out, in order. The results are then obtained in terms of BER vs. SNR plots. For validation of results, implementation in the same order is carried out on hardware. 
A. Modelling Doppler Shift

Doppler shift is modelled in terms of Doppler factor, whose values can range from 0 to 0.099. [19] describes mathematically how an OFDM symbol is affected by Doppler shift, assuming all paths in a multipath channel have same Doppler factor. As given in [19], each OFDM symbol on each path is scaled in duration. The duration changes from $\mathrm{T}$ to $\mathrm{T} /(1+\mathrm{a})$, where $\mathrm{a}$ is the Doppler scaling factor. Also, a frequency shift of $\mathrm{e}^{\mathrm{j} 2 \pi \mathrm{aft}}$ is experienced by each subcarrier. This shift is dependent on the frequency of the subcarrier and differs considerably between subcarriers because the bandwidth of the OFDM signal is comparable to the center frequency. Each subcarrier frequency $\mathrm{f}_{\mathrm{k}}$ is shifted by a factor $a f_{k}$. The new frequency of each subcarrier is equal to $f_{k}+a f_{k}$, i.e. $f_{k}(1+a)$. The orthogonality of subcarriers is thus lost and inter carrier interference (ICI) is present. The shift needs to be compensated to recover orthogonality. Since a frequency shift of $\mathrm{e}^{\mathrm{j} 2 \pi \mathrm{aft}}$ is experienced by each sub carrier, it is modelled along with the IDFT function. Each OFDM frame is given one Doppler scaling factor. The IDFT function is called for each OFDM symbol in a frame, with the value of the Doppler scaling factor to be applied passed as a parameter. In the function, the facto.r $\mathrm{e}^{\mathrm{j} 2 \pi \mathrm{aft}}$ is multiplied with the IDFT formula, thus incorporating it into transmitted time domain signal, as given in [19].

\section{B. Channel Transfer Function}

The behaviour of the channel is modelled using Thorp's attenuation profile, as described in [16]. Figure 1 shows the attenuation profile simulated on MATLAB. Before the spectrum is sent to the IDFT function, the complex 4 QAM symbols at each frequency are divided by the values of absorption coefficient at that frequency. The channel transfer function is the reciprocal of $\mathrm{A}(\mathrm{f})$. This transfer function is multiplied by the spectrum (also in the frequency domain) before sending to the IDFT function. In this manner, the attenuation provided by the ocean channel is modelled and taken into account in the OFDM system.

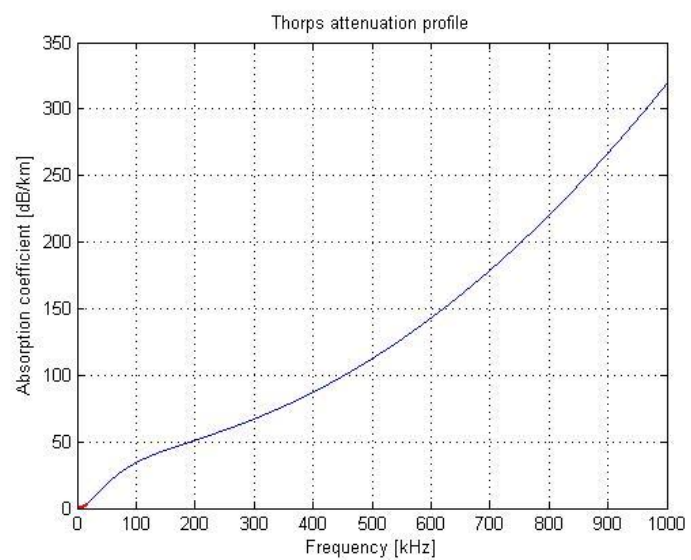

Figure 1. Thorp's attenuation profile

\section{Ambient Ocean Noise}

The four sources of ocean noise are modelled using equations given in [16]. Figure 2 shows the profiles of noise as simulated on Matlab. The values obtained in $\mathrm{dB}$ re $\mu \mathrm{Pa}$ per $\mathrm{Hz}$ are converted to absolute values and are then added to obtain the total ocean noise.

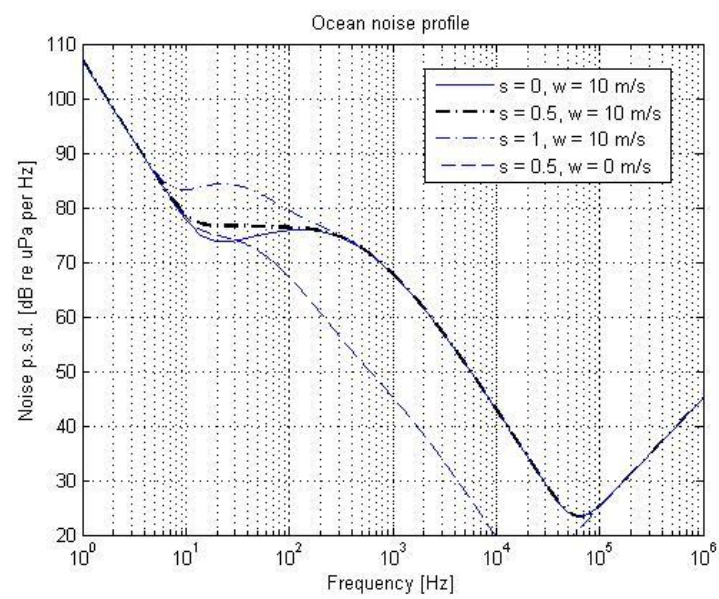

Figure 2. Total ambient ocean noise profiles for different shipping activities and wind speeds 
To add this noise to the QAM symbols in the spectrum, i.e. to the data the value must be obtained in watt. For this conversion, the specifications of a transducer are used. The transducer at the transmitter converts electric power to acoustic power. Therefore in terms of units, it converts $\mathrm{dB}$ to $\mathrm{dB}$ re $\mu \mathrm{Pa}$ per Hz. The conversion equation is modified since in this case, conversion is necessary in the reverse direction. The specifications of the transducer that are used are Transmitting Voltage Response (TVR), Sound Intensity Level (SIL) and its impedance. The unit of TVR is dB re $\mu \mathrm{Pa} / \mathrm{V}$ at $1 \mathrm{~m}$, and the unit of SIL is $\mathrm{dB}$ re $\mu \mathrm{Pa}$. The voltage level in $\mathrm{dB}, \mathrm{V}$ is given by

$$
\mathrm{V}=\mathrm{SIL}-\mathrm{TVR}
$$

Talking the antilog of this equation, the value of noise voltage $\mathrm{V}$ is obtained by dividing the value of total noise (whose $\log$ value is SIL) by the absolute value of TVR. The square of this voltage divided by impedance gives the power of the noise in watts. The noise power at each frequency is thus obtained and added to the QAM symbols at the respective frequencies in the spectrum, before it is sent to the IDFT function. Noise is modelled in this manner.

For each OFDM symbol (or frame), SNR is calculated as follows. One watt of signal power is given to each of the 16 tones, i.e. only the ones on which QAM symbols are transmitted. At each frequency, Thorp's absorption coefficient has a values $A(f)$ and noise power has a values $N(f)$. The SNR at each frequency is calculated as given in [16] as

$$
\mathrm{SNR}=1 /[\mathrm{A}(\mathrm{f}) \cdot \mathrm{N}(\mathrm{f})]
$$

The geometric mean of the 16 SNR values gives the SNR value of the OFDM symbol. SNR is varied by varying either signal power around values of 1 watt, or by varying noise power by changing values of wind speed and shipping activity.

\section{Implementation of Estimation and Compensation Algorithm}

The estimation and compensation algorithm is implemented at the receiver. The frames received are corrupted in that each is shifted by a different Doppler factor, is attenuated differently at each frequency and has a different amount of noise added at each frequency. From the stream being received, each frame is extracted as it comes. One frame consists of one OFDM symbol as preamble and 32 OFDM symbols containing data. For each frame, the preamble is extracted and used for the estimation algorithm, which is implemented as a separate block. This block returns the estimated value of Doppler shift for a particular frame. This estimated value, together with the 32 OFDM symbols that form data in a frame are sent to a compensation block. The compensation algorithm is implemented, the bit stream in then recovered and the BER value is calculated. The BER value is returned by the compensation block. Both the estimation and compensation blocks are shown in the receiver block diagram given in Figure 4.5.1. The transmitter is a standard OFDM transmitter as described previously.

A known copy of the uncorrupted preamble, i.e. the preamble generated at the transmitter, is shared with the estimation block in the receiver for every OFDM frame. The estimation block first extracts the shared preamble corresponding to the frame whose preamble it has been called with. It therefore works with an uncorrupted preamble shared by the transmitter and a received preamble corrupted by Doppler shift, absorption and noise.

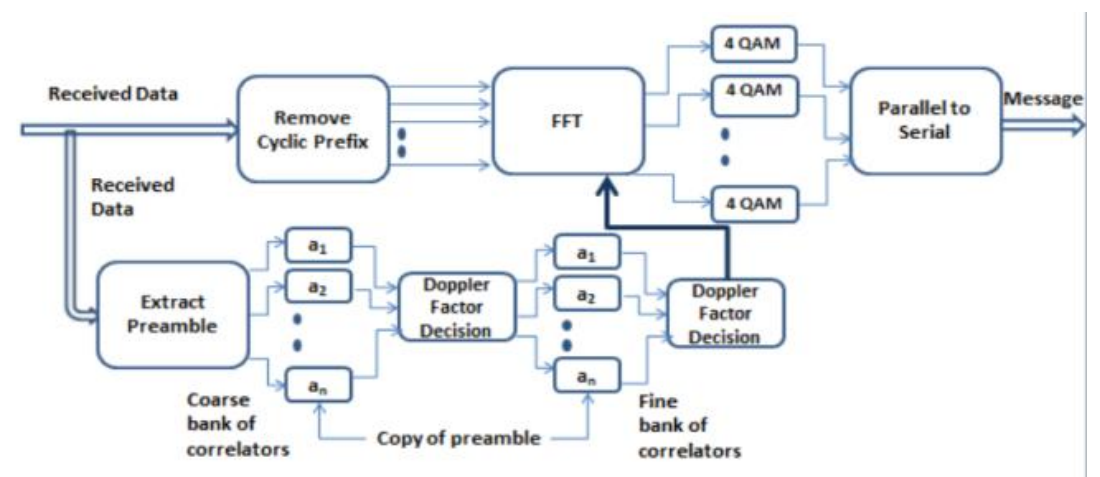

Figure 3. Receiver block diagram, with Doppler estimation and compensation blocks

The estimation process consists of two steps as shown in Figure 3: a coarse estimation, followed by a fine estimation. Both use a bank of correlators each. The coarse estimation block is called with the received preamble. For the coarse estimation, the values of Doppler factors associated with the correlators range from 0 to 0.09 , with a step size 0.01 . The coarse estimated value is returned by this block. The fine estimation block is called with both the received preamble and the coarse estimated value. For fine estimation, the correlators have a step size of 0.001 . The values of Doppler factors associated with the correlators are centred around the coarse estimated value. For example, for a coarse estimated value of 0.04 , the fine estimation bank of correlators will have Doppler factor values from 0.035 to 0.045 in steps of 0.001 . 
For both coarse and fine estimation, the shared preamble is in the form of a bit stream. It is passed through parallel QAM encoders followed by an IFFT block. For each correlator, the Doppler factor associated with that correlator is passed to the IFFT block. Each correlator therefore has a Doppler shifted copy of the shared preamble. The received preamble (which is not only Doppler shifted but also affected by noise and attenuation) is correlated with each of the stored preambles, using the correlation function. The one which gives the maximum value of correlation function determines the estimated value of Doppler factor.

The 32 OFDM symbols that constitute the data of a frame are sent to the compensation function along with the estimated value of Doppler factor. For each symbol the cyclic prefix is removed and DFT is taken. Since Doppler effect was modelled by multiplying the factor $\mathrm{e}^{\mathrm{j} 2 \pi \mathrm{aft}}$ in the IDFT formula, compensation is performed by multiplying the DFT formula by a factor $\mathrm{e}^{\mathrm{j} 2 \pi \mathrm{aft}}$. Therefore, when the DFT is taken for each symbol, the estimated Doppler scaling factor value is passed to the function, as indicated in Figure 3.

The compensation function then extracts the QAM symbols from the DFT spectrum obtained, and decodes them to obtain a bit stream. It compares this bit stream with the one transmitted, computes the BER and returns this value.

The complete system, together with modelling if channel, ambient ocean noise, estimation and compensation algorithm is indicated in Figure 4.

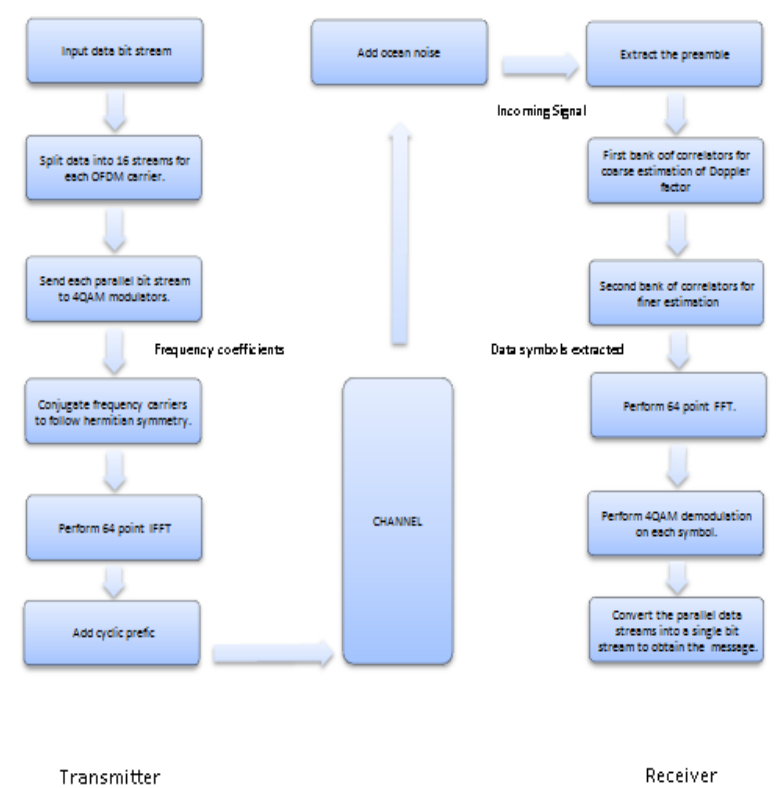

Figure 4. Flow chart of system

TABLE 1. SPECIFICATIONS OF THE SYSTEM

\begin{tabular}{|l|l|}
\hline Bandwidth & $0.5 \mathrm{kHz} \cdot 16.25 \mathrm{kHz}$ \\
\hline Number of subcarriers & 16 \\
\hline Modulation Scheme & $4 \cdot \mathrm{QAM}$ \\
\hline Cyclic prefix & $25 \%$ \\
\hline $\begin{array}{l}\text { Total number of bits per OFDM } \\
\text { symbol(inclusive of cyclic prefix) }\end{array}$ & 32 \\
\hline Symbols per frame & 32 (1024 bits in all) \\
\hline Preamble & 1 OFDM symbol \\
\hline IFFT/FFT Size & 64 \\
\hline
\end{tabular}

\section{RESULTS}

The estimation and compensation algorithms are validated by simulation on Matlab, by generating BER vs. SNR plots. Figure 5 shows the BER vs. SNR plot with no compensation, after coarse compensation and after fine compensation. The SNR is varied by varying signal power from 0.5 watt to 1 watt. Ambient ocean noise added is for shipping activity 1.5 and wind speed $15 \mathrm{~m} / \mathrm{s}$. Figure 6 shows the same plot only after coarse and fine estimation. 
Vol. 6, Issue 4, April 2017

It is observed that BER reduces with increase in SNR. Also, as compared to when no compensation is performed, BER reduces after coarse compensation is performed and reduces further after fine compensation is performed. For an SNR of $27.24 \mathrm{~dB}$, BER values are $0.5113,0.0141$ and 0.0004 with no compensation, coarse and fine compensation respectively. The BER thus reduces significantly from $10^{-2}$ to $10^{-4}$ after fine estimation and compensation.For an SNR of $26.27 \mathrm{~dB}$, BER values are $0.5125,0.0201$ and 0.003 with no compensation, coarse and fine compensation respectively. The BER thus reduces significantly from $10^{-2}$ to $10^{-3}$ after fine estimation and compensation.

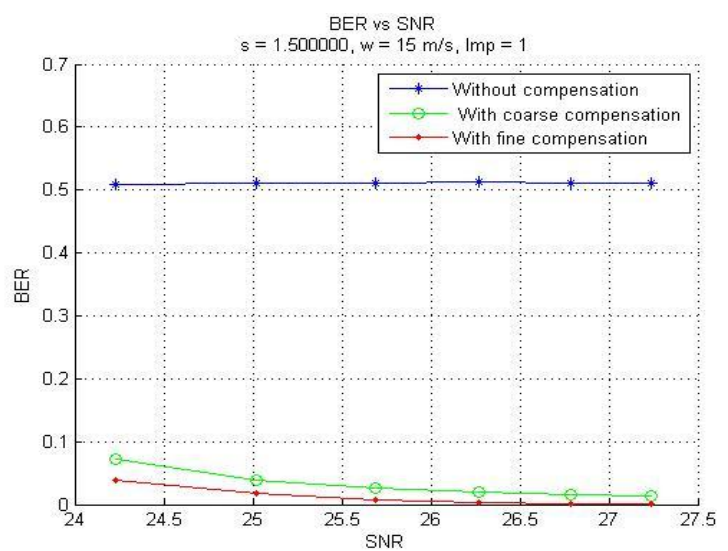

Figure 5. BER vs. SNR(in $\mathrm{dB}$ ) plot without compensation, after coarse and fine compensation for $\mathrm{s}=1.5 \mathrm{and} \mathrm{w}=15$ $\mathrm{m} / \mathrm{s}$

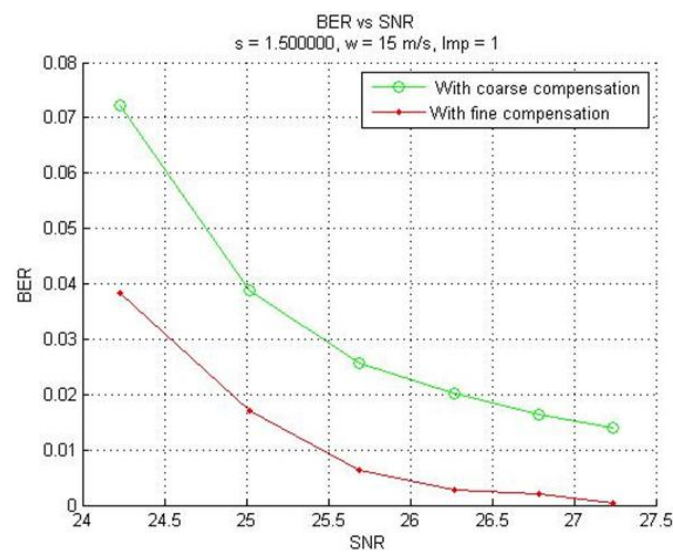

Figure 6. BER vs. SNR(in $\mathrm{dB}$ ) plot after coarse and fine compensation for $\mathrm{s}=1.5$ and $\mathrm{w}=15 \mathrm{~m} / \mathrm{s}$

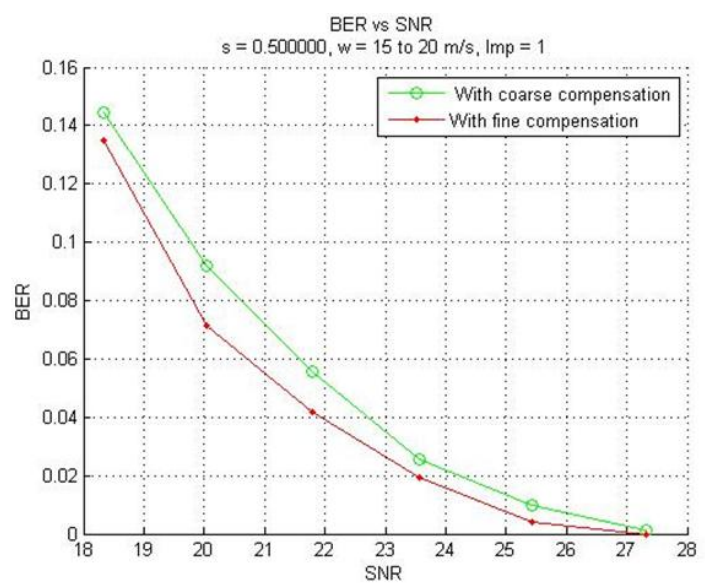

Figure 7. BER vs. SNR(in dB) plot after coarse and fine compensation for $\mathrm{w}=15 \mathrm{~m} / \mathrm{s}$ to $20 \mathrm{~m} / \mathrm{s}$

Figure 7 shows the BER vs. SNR plot after coarse compensation and after fine compensation. The SNR is varied by varying noise wind speed from $15 \mathrm{~m} / \mathrm{s}$ to $20 \mathrm{~m} / \mathrm{s}$. Signal power is 1 watt. 
Results of the coarse and fine estimation as obtained on the Zynq board, using Xilinx SDK, are shown below. Figure 8 shows the BER vs. SNR plots for a wind speed of $15 \mathrm{~m} / \mathrm{s}$ and shipping activity of 1.4 . The SNR is varied by varying the signal power around values of 1 watt.

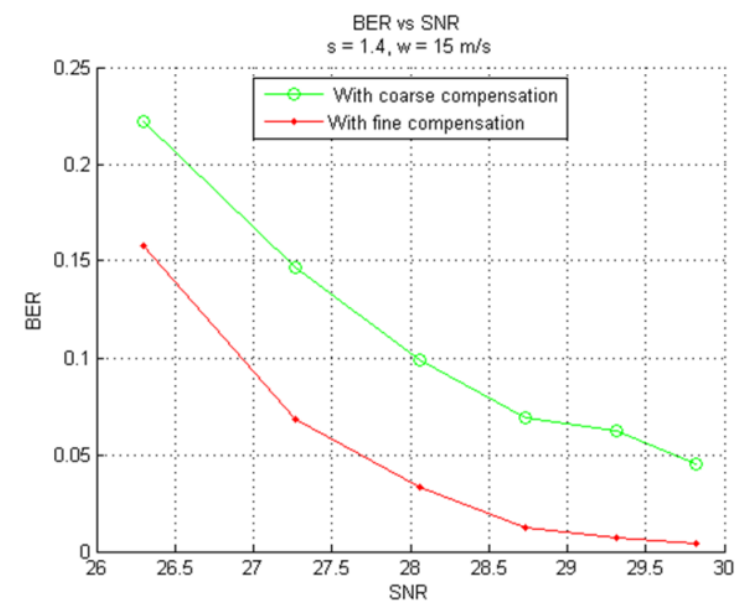

Figure 8. BER vs. SNR (in dB) plot for shipping activity 1.4 and wind speed $15 \mathrm{~m} / \mathrm{s}$

The reduction in BER after each stage of compensation is evident. For an SNR of $29.8 \mathrm{~dB}$, BER reduces from 0.4536 to 0.0455 to 0.0038 after each stage.

Figure 9 shows the BER vs. SNR plots for shipping activity of 1.2 and 1 watt power per tone. The SNR is varied by varying the wind speed from $8 \mathrm{~m} / \mathrm{s}$ to $15 \mathrm{~m} / \mathrm{s}$. As wind speed increases, noise power increases and thus SNR reduces. As SNR reduces, BER increases.

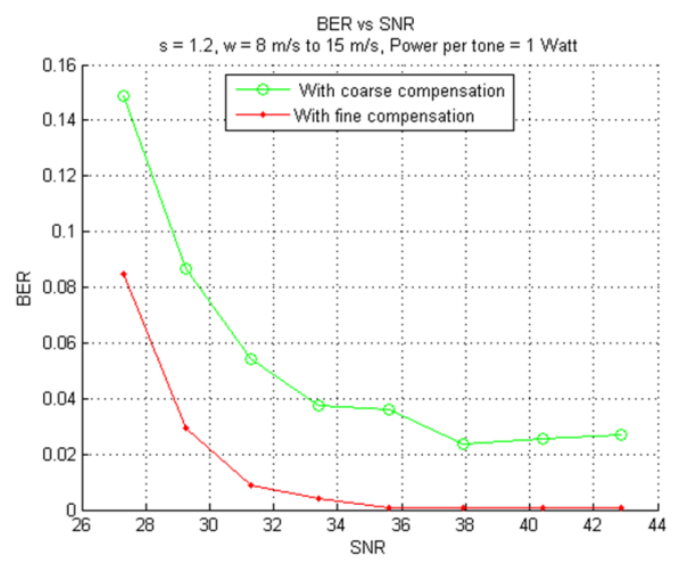

Figure 9. BER vs. SNR (in dB) plot for shipping activity 1.2, 1 watt power per tone and varying noise power

It is observed from Figure 9 that BER increases with increasing wind speed. For any wind speed, the value of BER reduces after coarse compensation and reduces further after fine compensation. For an SNR of $42.8 \mathrm{~dB}$, BER reduces from 0.4480 to 0.0269 to 0.0006 after each stage.

\section{FUTURE SCOPE}

Underwater Acoustic communication is widely gaining popularity these days but it has many drawbacks when compared to terrestrial communication. Certain issues such as ambient noise, attenuation and Doppler Effects were significantly resolved in this project but there is still room for further improvement. Also, other drawbacks like time varying multipath effects and short range of communication has not been taken into consideration in this project but can be focused on in the future.

The system designed in this project is for generic underwater communication. The algorithm can be modified for specific applications to improve the results. Some applications like Environmental monitoring, underwater exploration, underwater scientific data collection and military search and survey can have drastic leaps in advancement by designing application specific systems and developing the necessary algorithm. 


\section{CONCLUSION}

An extensive study on Underwater Acoustic Communication has been performed while working on this project. With this learning, a robust algorithm has been developed for the compensation and estimation of Doppler Effects which mitigates one of the major drawback and source of error. The Underwater acoustic channel was recreated on Matlab that includes the channel profile, Thorp's attenuation, Doppler Effect and Ambient Noise i.e. Shipping Noise, Wind speed, Turbulence and thermal noise. The simulation of the underwater communication was performed using the recreated channel and developing an OFDM transmitter and receiver. After implementing the algorithm on the system, positive results were obtained on Matlab. The BER v/s SNR plots showed that without compensation and estimation, the BER is around $50 \%$ but with coarse estimation and compensation of the Doppler factor a to the precision of $10^{-2}$ by using a bank of parallel self correlators, BER drastically reduces to below $3 \%$. The algorithm was further improved by using two banks of correlators and improving the precision to $10^{-3}$ and resulted in BER to be as low as under $0.02 \%$. Similar results were obtained by varying the SNR and various noises that were taken into consideration. The next step taken in the major project was the Hardware implementation of the algorithm. For this, the Zynq board SoC (ZC702) was used. The same algorithm was implemented on the board in C code with the help of Xilinx SDK. Similar results were obtained on the Zynq board and hence, the algorithm and results were successfully verified.

\section{REFERENCES}

[1] Byung-Chul Kim and I-Tai Lu, "Parameter study of OFDM underwater communications system," OCEANS 2000 MTS/IEEE Conference and Exhibition. Conference Proceedings (Cat. No.00CH37158), Providence, RI, 2000, pp. 1251-1255 vol.2.

[2] Johannes du Preez, "An Investigation into OFDM as a Suitable Modulation Technique for an Acoustic Underwater Modem", Stellenbosch University, December 2012

[3] S. Venkatesan and R. A. Valenzuela, "OFDM for 5G: Cyclic prefix versus zero postfix, and filtering versus windowing," 2016 IEEE International Conference on Communications (ICC), Kuala Lumpur, 2016, pp. 1-5

[4] Li Y, Stuber G, Orthogonal frequency division multi -plexing for wireless communications, Springer Press, New York, 2006

[5] W. K. Lam and R. F. Ormondroyd, "A novel broadband COFDM modulation scheme for robust communication over the underwater acoustic channel," IEEE Military Communications Conference. Proceedings. MILCOM 98 (Cat. No.98CH36201), Boston, MA, 1998, pp. 128-133 vol.1

[6] A. B. Salberg and A. Swami, "Doppler and frequency-offset synchronization in wideband OFDM," in IEEE Transactions on Wireless Communications, vol. 4, no. 6, pp. 2870-2881, Nov. 2005.

[7] M. Stojanovic, "Low Complexity OFDM Detector for Underwater Acoustic Channels," OCEANS 2006, Boston, MA, 2006, pp. 1-6

[8] Jianguo Huang, Jing Sun, Chengbing He, Xiaohong Shen and Qunfei Zhang, "High-speed underwater acoustic communication based on OFDM," 2005 IEEE International Symposium on Microwave, Antenna, Propagation and EMC Technologies for Wireless Communications, 2005, pp. 1135-1138 Vol. 2

[9] M. Sung, J. Lee and J. Jeong, "Localised discrete Fourier transform-spread M-ary amplitude shift keying orthogonal frequency division multiplexing with Hermitian symmetry for peak-toaverage power ratio reduction," in IET Communications, vol. 8, no. 11, pp. 1938-1946, July 242014

[10] M. Sung, S. Kang, J. Shim, J. Lee and J. Jeong, "DFT- Coherent Optical OFDM With Hermitian Symmetry for Fiber Nonlinearity Mitigation," in Journal of Lightwave Technology, vol. 30, no. 17, pp. 2757-2763, Sept.1, 2012

[11] M. Stojanovic and J. Preisig, "Underwater acoustic communication channels: Propagation models and statistical characterization," in IEEE Communications Magazine, vol. 47, no. 1, pp. 84-89, January 2009

[12] B. Li, S. Zhou, M. Stojanovic, Lee Freitag and P. Willett, "Non-Uniform Doppler Compensation for Zero-Padded OFDM over Fast-Varying Underwater Acoustic Channels," OCEANS 2007 - Europe, Aberdeen, 2007, pp. 1-6.

[13] K. Tu, T. M. Duman, J. G. Proakis and M. Stojanovic, "Cooperative MIMO-OFDM communications: Receiver design for Doppler-distorted underwater acoustic channels," 2010 Conference Record of the Forty Fourth Asilomar Conference on Signals, Systems and Computers, Pacific Grove, CA, 2010, pp. 1335-1339.

[14] M. Barbeau, S. Blouin, G. Cervera, J. Garcia-Alfaro, B. Hasannezhad and E. Kranakis, "Simulation of underwater communications with a colored noise approximation and mobility," 2015 IEEE 28th Canadian Conference on Electrical and Computer Engineering (CCECE), Halifax, NS, 2015, pp. 1532-1537.

[15] P.C. Etter, "Underwater Acoustic Modelling and Simulation", CRC Press, 2003

[16] M. Stojanovic, "On the Relationship Between Capacity and Distance in an Underwater Acoustic Communication Channel", ACM SIGMOBILE Mobile Computing and Communications Review (MC2R), vol. 11, no. 4, pp. 34-43, Oct. 2007.

[17] Y. Noguchi, Y. Kuranaga and T. Maki, "Adaptive navigation of a high speed autonomous underwater vehicle using low cost sensors for lowaltitude survey," 2017 IEEE Underwater Technology (UT), Busan, South Korea, 2017, pp. 1-4.

[18] Balas G.J., Bokor J., Vanek B., Arndt R.E. "Control of High-Speed Underwater Vehicles" In: Francis B.A., Smith M.C., Willems J.C. (eds), "Control of Uncertain Systems: Modelling, Approximation, and Design." Lecture Notes in Control and Information Science, vol 329. Springer, Berlin, Heidelberg, 2006

[19] B. Li, S. Zhou, M. Stojanovic, L. Freitag and P. Willett, "Multicarrier Communication Over Underwater Acoustic Channels With Nonuniform Doppler Shifts," in IEEE Journal of Oceanic Engineering, vol. 33, no. 2, pp. 198-209, April 2008

[20] J. G. Proakis, Digital Communications, NY: McGraw-Hill, 1995

[21] B. S. Sharif, J. Neasham, O. R. Hinton and A. E. Adams, "A computationally efficient Doppler compensation system for underwater acoustic communications," in IEEE Journal of Oceanic Engineering, vol. 25, no. 1, pp. 52-61, Jan. 2000

[22] S. F. Mason, C. R. Berger, S. Zhou and P. Willett, "Detection, Synchronization, and Doppler Scale Estimation with Multicarrier Waveforms in Underwater Acoustic Communication," in IEEE Journal on Selected Areas in Communications, vol. 26, no. 9, pp. 1638-1649, December 2008

[23] L. Ming, Z. Lin and Z. Yide, "A novel Doppler frequency shift compensation algorithm for OFDM underwater acoustic communication system," 2013 25th Chinese Control and Decision Conference (CCDC), Guiyang, 2013, pp. 1571-1575.

[24] Jie Yang, Xiangtian Liu and Fengqi Yu, "A Doppler shift estimation algorithm with low computational complexity for underwater acoustic communication," 2015 IEEE 16th International Conference on Communication Technology (ICCT), Hangzhou, 2015, pp. 47-50. 